Revista Complutense de Ciencias Veterinarias

ISSN-e 1988-2688

\title{
Efecto del estrés calórico y el estrés oxidativo en la función espermática de los mamíferos
}

\author{
Espinosa-Cervantes, Román y Córdova-Izquierdo, Alejandro ${ }^{1}$
}

\begin{abstract}
Resumen. Diversos estudios han demostrado una disminución en la capacidad reproductiva del macho por el estrés calórico debido a la producción de especies reactivas de oxigeno (ERO) en los espermatozoides. Por lo que el objetivo del presente trabajo es mostrar una visión general de los riesgos del ambiente cálido, el estrés oxidativo y los mecanismos por los cuales se presenta una alteración en la capacidad espermática en el macho. La mayor parte de las ERO, son producidas por los espermatozoides y leucocitos seminales. Las ERO causan daño en la membrana del espermatozoide (conteniene ácidos grasos poliinsaturados PUFAs), disminución de la motilidad, poca capacidad para fusionarse con el ovocito y alteración del ADN espermático, causando pobre desarrollo embrionario y baja fertilidad. Por lo que la determinación de ERO en espermatozoides animales podría ser una herramienta diagnóstica a tener en cuenta en la identificación de la etiología de la infertilidad del macho.
\end{abstract}

Palabras clave: espermatozoides, estrés calórico, estrés oxidativo, espermatozoides, ERO, mamíferos.

\section{[en] Effect of oxidative and thermal stress on sperm function in mammals}

\begin{abstract}
Several studies have reported a decrease in the reproductive capacity of the male due to heat stress and its effect on the production of reactive oxygen species (ROS) in sperm. Therefore, the objective of the present work is to show a general view of the risks of the warm environment, oxidative stress and the mechanisms by which there are an alteration in sperm capacity in the male. Sperm and seminal leukocytes produce most ROS. ROS cause damage to the sperm membrane (containing polyunsaturated fatty acids PUFAs), decreased motility, poor ability to fuse with the oocyte, and alteration of sperm DNA, causing poor embryonic development and low fertility. Therefore, the determination of ROS in sperm could be considered an alternative diagnostic tool to identify the etiology of male infertility.
\end{abstract}

Key words: sperm, thermal stress, oxidative stress, spermatozoa, ROS, mammalian.

\section{Introducción}

Recientemente, diversos estudios han mostrado una disminución en la capacidad reproductiva de los animales domésticos y de vida salvaje. Los efectos en la reproducción son debidos a condiciones ambientales (calor), con efectos económicos negativos y consecuencias dramáticas en la producción de alimentos (Chirault et al. 2015).

La mayor parte de los tejidos, tipos celulares, señales metabólicas y reacciones bioquímicas se ven afectados en mayor o menor medida por el estrés calórico. Sin embargo, hay algunos tipos celulares especialmente termo sensibles como las células germinales masculinas de los mamíferos (Salce-Ortiz et al. 2015). La exposición al calor de los animales domésticos resulta en un incremento de la temperatura escrotal en los machos y puede verse afectada por una diversidad de factores externos como la postura, la época del año y la desregulación de factores térmicos, los cuales han sido implicados en la patogénesis y la infertilidad del macho (Nezhad et al. 2013). Por otra parte, también se han considerado algunas otras causas que afectan la temperatura escrotal como el criptorquidismo, el varicocele y la fiebre crónica (Palmer et al. 2012).

Aunque los testículos están suspendidos por el escroto fuera del cuerpo en algunas especies animales (verraco, toro, borrego y humano), la 
espermatogénesis puede verse alterada por la exposición a altas temperaturas debilitando la capacidad del sistema de termorregulación al inducir una mayor temperatura en los testículos provocando una disminución en la calidad de semen en el cerdo, toro, ratón y el humano (Wechalekar et al., 2010; Nezhad et al. 2013). Esto es debido en parte a que la mayor parte de células del testículo, son células de Sertoli y que gran parte de ellas se ven alteradas como resultado del estrés calórico, que resulta deletéreo en dichas células. Por lo que la determinación del número células de Sertoli mediante la proteína de unión a andrógenos, inhibina $\mathrm{B}$, factor inhibidor de Mülleriano y otros polipéptidos que constituyen marcadores de la función de la célula de Sertoli vivas sería un buen criterio de evaluación del impacto perjudicial del estrés calórico en el testículo (Nezhad et al. 2013).

Estudios recientes han reportado que el estrés calórico escrotal induce estrés oxidativo severo en algunas especies animales lo cual causa muerte de las células germinales ( $\mathrm{Li}$ et al. 2013). El estrés oxidativo puede conducir a la infertilidad por medio de dos mecanismos: en primer lugar se produce un daño en los lípidos y proteínas la membrana del espermatozoide, provocando una reducción de la motilidad y de la capacidad de fusionarse con el ovocito $\mathrm{y}$, en segundo lugar, daña el DNA del esperma que disminuye el desarrollo embrionario temprano, aumenta la incidencia de aborto involuntario y reduce la fertilidad en animales domésticos (Aitken et al. 1998; Aitken y De Luliis, 2010; Tremellen, 2008).

Con el inicio de la reproducción asistida hace aproximadamente un cuarto de siglo, surgió la necesidad desarrollar técnicas sofisticadas para separar espermatozoides por motilidad, viabilidad y características morfológicas, como piedra angular en la evaluación de la fertilidad animal, aunque las tasas de éxito de estos procedimientos siguen siendo subóptimas (Paasch et al. 2007). Inicialmente las técnicas de separación consistían en un simple lavado; técnicas de separación basadas en diferentes principios como migración, filtración o centrifugación en gradientes de densidad, para separar espermatozoides funcionales, capaces de moverse y con capacidad de fecundar ovocitos (Vasan, 2011). Sin embargo, estas técnicas tienen limitaciones que las hacen no siempre predecir con exactitud la fertilidad. Las técnicas de análisis seminal necesitan ser completadas con pruebas funcionales, las cuales indirectamente miden la habilidad de que un espermatozoide esté en buenas condiciones para fecundar un ovocito. Se ha descrito que existe una asociación muy importante entre calidad espermática y el estrés oxidativo, en los mamíferos, pero rara vez se piensa en tratar el estrés oxidativo y dar un tratamiento para mejorar esta condición. Recientemente se ha visto que la selección de los espermatozoides depende de la calidad del eyaculado y esto incluye la determinación de especies reactivas de oxigeno (ERO), en los espermatozoides y en los leucocitos (Henkel and Schill, 2003; Oborna et al., 2009; Vasan, 2011; Alves et al. 2015). El método de quimioluminiscencia es la técnica más comúnmente empleada como una medición directa de la generación de ERO por parte de los espermatozoides. Este ensayo es capaz de cuantificar ERO tanto intracelulares como extracelulares (Kashou et al. 2013).

Por lo que el objetivo del presente trabajo es mostrar una visión general de los riesgos de la temperatura ambiental elevada, el estrés oxidativo y los mecanismos por los cuales se produce la infertilidad en el macho.

\section{Efecto de la temperatura en los testículos}

La literatura internacional ha reportado que la elevación de la temperatura intratesticular tiene un efecto adverso sobre la espermatogénesis, provocando la alteración de los parámetros espermáticos y la apoptosis (Jung y Schuppe, 2007). Las causas principales de esta alteración son la formación de ERO que proveen cambios moleculares en el ADN, proteínas, lípidos y otras moléculas biológicas que contribuyen a la baja fertilidad en los animales (Shekarriz et al. 1995; Jung y Schuppe, 2007; El-Tohamy, 2012). Investigaciones previas usando modelos animales, han sugerido que las alteraciones en las espermatogénesis debidas a la exposición de los testículos a altas temperaturas provocan un incremento en el metabolismo, causando en el testículo una alta demanda de oxígeno. Por lo que el flujo sanguíneo del testículo será naturalmente bajo y por consiguiente causará estrés hipóxico en las células del parénquima activando el proceso de degeneración testicular (Galil y Setchell, 1988; Setchell, 2006; Ramires et al. 2013). El estrés calórico en los testículos de los bovinos altera él porcentaje de motilidad espermática 
al largo de las estaciones del año (39.15 \pm 1.98 , $41.25 \pm 1.79,33.80 \pm 2.06$ y $37.30 \pm 2.02$, en invierno, primavera, verano y otoño, respectivamente). Siendo el verano la estación del año con mayor temperatura ambiental y menor motilidad espermática (Perumal et al. 2017). En bovinos (Braford) criados en regiones tropicales, los días calurosos aumentan la temperatura testicular y pone en riesgo la espermatogénesis y la calidad seminal. Esto debido a que las altas temperaturas interfieren con el metabolismo oxidativo de la glucosa en las células espermáticas como resultado de la disfunción mitocondrial y la generación de ERO (Oliveira et al. 2014; Hamilton et al. 2016).

En otro estudio se determino la influencia de la estación en la obtención de semen en toros Bos taurus (Simmental) y concluyo que el estrés calórico del verano afectó los parámetros de calidad y esto podría estar relacionado con la alta peroxidación de lípidos. También se ha observado que cuando se obtiene semen de toros jóvenes en verano hay un daño oxidativo más intenso de las proteínas (Balic et al. 2012). Los aminoácidos contienen puentes disulfuro y grupos tiol que son sensibles al estrés oxidativo lo que conduce a la escisión de la cadena de proteínas (Jahanian et al. 2014).

En otras especies como en porcino también se ha demostrado que cuando son expuestos a estrés calórico a temperaturas en torno a $42{ }^{\circ} \mathrm{C}$ durante seis horas, se inducen cambios importantes en la motilidad espermática, disminuyéndola hasta en un 32\% (Gong et al. 2017). En el ratón la hipertermia testicular transitoria también causa muerte de las células germinales y demuestra que temperaturas elevadas (40 a $42{ }^{\circ} \mathrm{C}$ ), provocan estrés y por consecuencia la inducción de genes asociados con el estrés oxidativo y la hipoxia (Catriona et al. 2009).

Existen otras causas que afectan la espermatogénesis y la calidad espermática como son los trastornos clínicos tales como criptorquidia y el varicocele que están implicados en la fisiopatología de la espermatogénesis, mediante la alteración del flujo de sangre testicular, la hipertermia y en consecuencia estrés oxidativo e hipoxia (Mieusset et al. 1987; Fretz y Sandlow, 2002). Existen evidencias de que el calentamiento escrotal activa las respuestas del estrés testiculares al igual que en otros tejidos tales como: (a) la expresión de la subunidad $\alpha$ del factor inducible por hipoxia 1 (HIF-1 $\alpha$ ), un factor de transcripción cuyos genes diana están involucrados en la vasodilata- ción, la angiogénesis y la glicólisis (Shweiki et al. 1992; Wenger, 2002); (b) el HIF-1 $\alpha$ induce la expresión génica del factor de crecimiento del endotelial vascular (VEGF), un potente estimulador de la angiogénesis y del crecimiento endotelial (Schweiki et al. 1992; Forsythe et al. 1996; Paul et al. 2009), actuando a través de la interacción con sus respectivos receptores: el dominio de inserción de cinasa (KDR) y la tirosina cinasa tipo fms 1 (FLT1) (Ferrara, 2004) y, (c) la activación de las proteínas de choque térmico (HSP), que son a su vez reguladas por factores de choque térmico (HSFs) (Feder y Hofmann, 1999). Las HSPs se describen como chaperoninas con funciones de protección y antiapoptóticas, involucradas en el proceso de asistencia en el plegamiento correcto de proteínas mal plegadas por el estrés oxidativo y la prevención de su agregación en respuesta a la tensión térmica y oxidativa. Las HSP han sido identificadas por inmunofluorescencia en la superficie del esperma del ratón, rata, toro, verraco y al parecer son accesibles para el marcado de las proteínas de la superficie espermática con biotina y yodo radiactivo (Naaby-Hansen y Herr, 2010).

Otro factor que tiene un papel importante en la regulación de la temperatura escrotal ha sido estudiado mediante el efecto de la energía en la dieta y la raza, en bovinos Angus, Simmental x Angus y Simmental, o Herford $\mathrm{x}$ Simmental, en donde se ha descrito que la dieta puede afectar la termorregulación escrotal o testicular mediante la reducción de la cantidad de calor que puede ser radiada desde el cuello del escroto. Diferencias de $0.5^{\circ} \mathrm{C}$, afectan a la calidad espermática (Coulte et al. 1988). Además, se ha observado que los animales alimentados con dietas moderadas en energía (100\% forraje; $9.110 \mathrm{MJ} / \mathrm{kg}$ ) tuvieron un $52 \%$ más de reservas espermáticas en el epidídimo y un 12 $\%$ más de producción diaria de espermatozoides en comparación con los toros alimentados con dietas altas en energía $(80 \%$ grano $+20 \%$ forraje; $13.064 \mathrm{MJ} / \mathrm{kg}$ ). También se ha descrito que los cruzamientos de Simmental tuvieron más reservas espermáticas en epidídimo 29.9 \pm 3.6 que la raza Angus 18.4 \pm 2.2 (Coulter et al. 1997). Otro estudio similar realizado con bovinos Bos indicus y Bos taurus también muestra que la disminución de la calidad espermática está asociada con la temperatura de la superficie escrotal y la longitud del cuello escrotal (Brito et al. 2004). Estos mismos autores mencionan que los Bos indicus mostraron 
una reducción de la temperatura en la superficie escrotal y por consecuencia mejor calidad de semen con respecto a la motilidad y la morfología normal de los espermatozoides.

Algunas de las posibles explicaciones es que la raza de toros Bos indicus tiene una arteria testicular con mayor longitud en proporción al volumen testicular y un menor espesor en la pared de la arteria testicular que pueden ser responsables de un mayor enfriamiento de sangre arterial en el cordón espermático comparado con el Bos taurus (Brito et al. 2004; Setchell, 2006; Oliveira et al. 2014).

\section{Las especies reactivas de oxígeno y la peroxidación de lípidos de la membrana plasmática del espermatozoide}

El proceso de lipoperoxidación (LPO) en los espermatozoides ocurre durante el proceso de almacenamiento (congelación y descongelación) y el estrés calórico. Durante estos eventos se produce estrés oxidativo, que conduce a la LPO de la membrana de los espermatozoides, resultando en una mayor permeabilidad, pérdida de la integridad de la membrana y reducción intracelular de enzimas antioxidantes. Causando una mayor susceptibilidad al daño oxidativo (Ross et al. 2010; Kadirvel et al. 2014).

Es conocido que los espermatozoides de los mamíferos son susceptibles a perder la motilidad debido a los oxidantes endógenos como una consecuencia de la LPO, que culmina en la generación de aldehídos citotóxicos como el malondialdehido y 4-hidroxinonenal (Aitken et al. 2012). En un estudio, se observó que los niveles de aldehídos registrados después de la adición de un ión ferroso a los espermatozoides, estaba altamente correlacionado con la competencia funcional de estas células, ya que redujo la motilidad, la viabilidad, la capacidad de los espermatozoides para una prolongada supervivencia in vitro y la competencia del esperma para fusionarse con el ovocito (Aitken y Curry, 2011; Aitken et al. 2012).

La susceptibilidad de los espermatozoides de los rumiantes, al estrés oxidativo es una consecuencia de la abundancia de ácidos grasos poliinsaturados (PUFAs) en la membrana plasmática de los espermatozoides, son sensitivos al daño inducido por el oxígeno mediado por la LPO y la rápida pérdida de ATP que conduce al daño del axonema, principal pie- za motora del flagelo de los espermatozoides y por lo tanto la motilidad espermática se ve disminuida (Bansal y Bilaspuri, 2010; Hamilton et al. 2016). Los PUFAs le proporcionan fluidez y flexibilidad a la membrana ayudando al espermatozoide en el acoplamiento en los eventos de fusión membranal durante la fecundación. Desafortunadamente, la presencia de dobles enlaces en estas moléculas las hace vulnerables al ataque de los radicales libres y a la iniciación de la cascada de LPO. Dando como resultado la pérdida de la membrana y la integridad morfológica, dañando las funciones celulares y la motilidad espermática (Bansal and Bilaspuri, 2010; Bucak et al. 2009; Jahanian et al. 2014). La vitamina $E$ es un antioxidante extracelular y es capaz de disminuir los efectos negativos sobre los PUFAs de los espermatozoides de los mamifieros, dicha vitamina es utilizada como suplemento alimenticio (Abavisani et al. 2013).

En algunas especies animales como el macho cabrio se ha observado que la producción espermática disminuye con el estrés calórico y es atribuido a la interacción directa que ejerce el estrés calórico y las ERO a la membrana de las células espermáticas. Lo cual resulta en el deterioro de la fluidez, la permeabilidad de la membrana y el daño de las células germinales y los espermatozoides maduros (El-Tohamy et al. 2012a). Kadirvel et al. (2014), estudiaron la actividad enzimática antioxidante y su relación con la LPO en semen de Búfalo (Bubalus bubalis), los resultados mostraron una disminución en la actividad de la super oxido dismutasa (SOD), Glutation peroxidasa (GSHPx) y glutatión (GSH). Estas enzimas forman parte del sistema antioxidante de la célula, y reaccionan contra los hidroperóxidos contribuyendo a la protección de los espermatozoides del daño oxidativo. La actividad de estas enzimas tuvo fuerte asociación con motilidad, integridad de la membrana y una correlación negativa con las ERO y la LPO.

Se sabe que la albúmina sérica bovina (BSA) puede sustituir a la yema de huevo en el diluyente del semen de carnero, para eliminar los radicales libres generados por el estrés oxidativo y protegiendo así la integridad de la membrana de los espermatozoides del choque térmico durante la congelación-descongelación del semen canino. Los carotenoides como beta-caroteno y licopeno también son componentes importantes de la defensa antioxidante 
contra la peroxidación de lípidos de los espermatozoides de la rata (Uysal y Bucak, 2007).

Para prevenir la LPO en los espermatozoides como consecuencia del estrés calórico, se debe hacer un esfuerzo para desarrollar combinaciones óptimas de antioxidantes en la alimentación de los animales que refuercen la defensa antioxidante, y así prevenir la excesiva generación de ERO en los espermatozoides que esta principalmente inducida por diversos factores como: el desacoplamiento de la fosforilación oxidativa en la mitocondria, el ciclo Redox de los xenobioticos y la excesiva actividad de NADPH oxidasa. Además las deficiencias de la dieta, la edad o los factores genéticos son factores predisponentes (Aitken et al. 1998).

\section{Papel fisiológico de las especies reactivas de oxígeno en el sistema reproductivo del macho}

Las ERO presentan efectos benéficos y dañinos en la función espermática, dependiendo de la naturaleza, la concentración, localización y el tiempo de exposición (Chandra et al. 2012). Durante su camino por el epidídimo el espermatozoide adquiere la habilidad de moverse progresivamente. Sin embargo, ellos adquieren la habilidad para fecundar, en el tracto de la hembra a través de una serie de cambios fisiológicos conocidos como "capacitación", que comprende cambios en la membrana plasmática de los espermatozoides necesarios para que se fusione con él ovocito. Durante este proceso, los niveles de calcio intracelular, las ERO y la tirosina cinasa aumentan, dando lugar a un aumento de Monofosfato de Adenosina cíclico (AMPc). El aumento de AMPc facilita la hiperactivación de los espermatozoides condición por la cual son altamente móviles (Aitken, 1995). En condiciones fisiológicas, los espermatozoides producen pequeñas concentraciones de ERO, las cuales son necesarias para la capacitación y la reacción acrosomal, hiperactivación, motilidad y fertilización (Agarwal et al. 2004; Agarwal y Sekhon, 2010).

En un estudio se reportó que co-incubando espermatozoides con bajas concentraciones de $\mathrm{H}_{2} \mathrm{O}_{2}$ (peróxido de hidrógeno), se estimuló la capacitación espermática, la hiperactivación y la fusión con el ovocito (Griveau et al. 1994). Estos autores confirmaron lo anterior con la adición de catalasa al inicio del proceso de ca- pacitación y reportaron la disminución de hiperactivación e inducción de la reacción acrosomal normal. De acuerdo con otro estudio, el anión superóxido y la generación de oxido nítrico durante la capacitación de los espematozoides también toman parte en estos procesos (Francavilla et al. 2000). Además, la bajas concentraciones de peroxido de hidrógeno causa fosforilación de tirosina, (este efecto parece ser general en todas las especies de mamíferos incluyendo al hombre), la cual resulta en la unión de las proteínas de la membrana del espermatozoide con las glicoproteínas ZP-3 en la zona pelúcida y posteriormente la fusión del espermatozoide con el ovocito (Aitken et al. 1995; Makker et al. 2009).

Los embriones producidos por fecundación de ovocitos de la raza Brahman con semen de Angus fueron más resistentes a choque térmico que los embriones producidos por fecundación de los ovocitos de Holstein con semen Angus (Block et al. 2002). En el mismo experimento, no hubo diferencias en la termotolerancia de embriones de Brahman x Holstein y Angus x Holstein. Una explicación para este fenómeno es que los transcritos acumulados en el ovocito son los responsables de la termotolerancia y que las diferencias en la expresión génica es probable que ocurran en las primeras etapas del desarrollo (Hansen, 2013).

Además, las ERO también juegan un papel importante en la compactación de la cromatina espermática durante la maduración del espermatozoide a lo largo del epidídimo, formándose puentes disulfuro que le otorgan al núcleo espermático una estructura altamente compacta. Esta proteína lleva un extremo N-terminal como señal única de localización nuclear, lo que le permite desempeñar un doble papel, facilitar condensación espermática de la cromatina y al mismo tiempo proporcionar protección al ADN contra el daño oxidativo (Pfeifer et al. 2001; Aitken et al. 2012).

\section{Fuentes de especies reactivas de oxígeno en el semen}

Las fuentes principales de ERO en los espermatozoides son: células germinales inmaduras, las que presentan alguna anormalidad morfológica, células epiteliales, espermatozoides con residuos citoplasmáticos y las producidas por contaminaciones con leucocitos (leucocitoespermia $>10^{6}$ leucocitos $/ \mathrm{ml}$ de semen), 
particularmente neutrofilos y macrófagos (Jahanian et al. 2014). Los leucocitos activados son una fuente muy importante de ERO. Fisiológicamente producen hasta 1000 veces más ERO que los mismos espermatozoides, debido a la necesidad fisiológica de liberar grandes cantidades de superóxido para el ataque de patógenos, lo que desencadena una gran toxicidad hacia los espermatozoides (Ford, 2004; Henkel, 2011). Además, existe una correlación positiva entre la producción de ERO y citocinas proinflamatorias en el plasma seminal, tales como Interleucina IL-, IL-8 y Factor de necrosis tumoral- $\alpha$ (TNF- $\alpha$ ) (Tremellen, 2008).

Otra fuente principal de ERO es la reducción de oxígeno por el sistema Redox (reacciones de reducción y oxidación), de la membrana plasmática, destinado a reducir el potencial de transporte a través de la membrana plasmática de la célula (de Grey, 2003). La generación de ERO por los espermatozoides ocurre mediante dos vías: $i$.) el sistema Nicotinamida Adenina Dinucleótido Fosfato (NADPH)-oxidasa, a nivel de la membrana plasmática de los espermatozoides, que genera anión superoxido $\mathrm{y}$, ii.) la oxido-reductasa NADPH-dependiente a nivel mitocondrial (Makker et al. 2009).

El análisis de semen es un componente esencial en la fertilidad del macho. Para su estudio existen técnicas: manuales, automáticas y asistidas por computadora (sistema CASA para análisis de esperma automático). Una parte inherente dentro de estos estudios es la determinación de leucocitos mediante Espectroscopia de Resonancia Paramagnética Electrónica que puede detectar concentraciones superóxido generadas hasta por 2000 leucocitos activados $/ \mathrm{ml}$. Además, existen otros métodos de detección de ERO por luminiscencia e inmunocitoquímica (Ford, 2004). También, los leucocitos puedes ser removidos de la muestra espermática usando perlas de magnéticas recubiertas con anticuerpos contra el antígeno leucocitario común CD45, frecuentemente combinado con anti-CD15 para una mayor eficacia contra neutrófilos y monocitos (Richer y Ford, 2001; Ford, 2004).

\section{Estrés oxidativo en espermatozoides}

La gran paradoja de la respiración aeróbica es que el oxígeno, es esencial para la producción de energía en muchos tipos de células incluyendo los gametos. Sin embargo, también es dañino debido a que produce ERO, en los espermatozoides cuando son manipulados en condiciones in vitro durante las técnicas de reproducción asistida, estas células corren el riesgo de generar y ser expuestas a altos niveles de ERO (Du Plessis et al. 2008).

En investigaciones anteriores, no había pruebas de que el sexado de espermatozoides tenía cualidades de hiperactivación, una característica de la capacitación que podría promover la fecundación del ovocito. Además, en ese mismo estudio, se demostró de que la motilidad espermática del semen de la raza cebú (Bos indicus) tuvo un efecto negativo menor por el proceso de sexado en comparación con el semen sexado de toros Bos taurus (López et al. 2015).

Durante el proceso de sexado de espermatozoides con citometría de flujo se provoca un estrés en los espermatozoides que causa un aumento en la peroxidación de lípidos de la membrana. La defensa natural contra la oxidación proporcionada por plasma seminal se pierde por la alta dilución que hay que realizar durante la clasificación. Esto puede ser más evidente cuando los espermatozoides son procesados para su almacenamiento en nitrógeno líquido que aumenta la peroxidación de lípidos de la membrana. La manipulación de espermatozoides como el enfriamiento, preservación líquida a largo plazo y su congelación causan daños en la estructura y a fisiología y por consecuencia la LPO (Castro et al. 2016; Hu et al. 2016). Una de las principales razones en la reducción de la supervivencia de los espermatozoides es la presencia de ERO. Las ERO son conocidas por causar una disminución de la motilidad e inducir pre- capacitación, así como dañar al sistema de membrana por oxidación de los lípidos, especialmente cuando el contenido de plasma seminal se reduce después de extensa de dilución y lavado de los espermatozoides. Varios pasos en el proceso de clasificación de los espermatozoides llevan a la producción de las ERO, pero su efecto negativo sobre la peroxidación de lípidos se puede minimizar por la suplementación de medio con sustancias antioxidantes como con el pretratamiento espermático con glutatión (GSH) que mantiene la viabilidad y la fertilidad al reducir la apoptosis mejorando el desarrollo embrionario (Espinosa y Córdova, 2013; Hu et al. 2016).

La espermatogénesis es un proceso sumamente activo, capaz de generar aproximadamente 1.000 espermatozoides por segundo. 
Las altas tasas de división celular inherente a este proceso implican correspondientemente altas tasas de consumo de oxígeno mitocondrial por el epitelio germinal. Sin embargo, la vascularización de los testículos es reducida lo que provoca que la concentración de oxígeno en este tejido sea baja. Por lo que la baja concentración de oxígeno que caracteriza a este tejido, podría ser un componente importante del mecanismo por el cual el testículo se protege de los daños que causan los radicales libres (Aitken and Roman, 2008).

Estudios previos han demostrado que las células espermáticas tienen menor frecuencia de mutaciones en comparación con las células somáticas. Sin embargo, las células germinales están continuamente afectadas de forma negativa por factores endógenos y exógenos como las ERO afectando al ADN (Celino et al. 2011). También, se menciona que los espermatozoides son altamente sensibles a daños inducidos por las ERO, mientras que las espermatogonias son consideradas como tolerantes a las ERO. Se ha descrito que cuando se exponen ratones a estrés calórico leve puede provocar estrés oxidativo, con un gran numero de células germinales apoptóticas, mientras que en las espermatogonias la apoptosis es poco frecuente (Paul et al. 2009).

\section{Efecto de las especies reactivas de oxígeno en la motilidad espermática}

La patología que subyace detrás de la capacidad de las ERO para reducir la motilidad del esperma fue informada por Jones et al. (1979), él menciona que las ERO inducen peroxidación de la membrana de esperma, disminuyendo la flexibilidad y por lo tanto el movimiento de la cola. La membrana del esperma es vulnerable a este tipo de daño, ya que contienen grandes cantidades de ácidos grasos insaturados. Las ERO dañan directamente a las mitocondrias, disminuyendo la energía disponible e impidiendo la movilidad del espermatozoide. Por cualquier mecanismo, el estrés oxidativo afecta la motilidad del esperma y como resultado una menor cantidad de esperma alcanzaran el ovocito para la fecundación (Kao et al. 2008; Tremellen, 2008).

El vínculo entre las ERO y su efecto sobre la motilidad espermática es debido a una cascada de eventos que resultan en una disminución en la fosforilación de las proteínas del axonema y por consecuencia la inmovilización del espermatozoide, ambos procesos están asociados con la reducción en la fluidez de membrana (El-Tohamy, 2012). Otra hipótesis es que el $\mathrm{H}_{2} \mathrm{O}_{2}$ puede difundirse a través de las membranas en las células e inhibir la actividad de algunas enzimas tales como la glucosa6-fosfato-deshidrogenasa (G6PD). Esta enzima controla la velocidad de flujo de la glucosa a través de la vía hexosa monofosfato que a su vez, controla la disponibilidad intracelular de fosfato de dinucleótido de nicotinamida y adenina (NADPH). Esto a su vez es utilizado como una fuente de electrones por los espermatozoides para la generación de la ERO por un sistema de enzima conocida como la NADPH oxidasa (Aitken, 1997). La inhibición de la G6PD conduce a una disminución en la disponibilidad de NADPH y una acumulación concomitante de glutatión oxidado y glutatión reducido. Esto puede reducir las defensas antioxidantes de los espermatozoides y aumentar la peroxidación de los fosfolípidos de la membrana (El-Tohamy, 2012).

En algunos estudios se ha propuesto que las ERO afectan negativamente la función espermática, tanto in vivo como in vitro. Como es el caso de las técnicas de preparación de espermas para las tecnologías de reproducción asistida (TRA), son causas potenciales para la producción de ERO. Algunos estudios han relacionado la concentración de $\mathrm{H}_{2} \mathrm{O}_{2}$ exógeno con los parámetros de motilidad espermática de los niveles de las ERO y de óxido nítrico (NO) para reiterar la importancia de reducir al mínimo los niveles de ERO en TRA. Los resultados mostraron que el $\mathrm{H}_{2} \mathrm{O}_{2}$ afectó los parámetros del esperma. $\mathrm{El}_{2} \mathrm{O}_{2}$ fue perjudicial para la motilidad y resultó en un aumento significativo en la generación de ERO y los niveles de NO. Además, se observo un aumento significativo en células estáticas. Aunque este experimento demostró la necesidad de reducir los niveles de ERO exógeno durante TRA, no ilustran la relación causa-efecto de las ERO intracelulares y los niveles de NO con la motilidad de los espermatozoides. Por lo que es de vital importancia realizar investigaciones adicionales para definir un nivel patológico de las ERO (Du Plessis et al. 2010).

Para confirmar los efectos negativos de las ERO en la motilidad espermática en Brasil, se llevó a cabo un estudio bajo condiciones tropicales con toros Bradford (5/8 Hereford x 3/8 Nelore), en donde se evaluó el efecto 
estacional del medio ambiente en la calidad espermática de los sementales. Este estudio mostró que el gradiente de temperatura $(0,9$ ${ }^{\circ} \mathrm{C}$ ), tuvo una correlación positiva entre motilidad y vigor (espermático), con valores 0,36 y 0,35 respectivamente. Mientras que el promedio de motilidad masal fue de $(2,58)$ y una motilidad de $(52,64 \%)$ y un vigor de $(2,7)$ del semen disminuyo en el verano cuando fue comparado con otras estaciones (Oliveira et al. 2014). En otro estudio Coulther (1988), reporto una motilidad espermática del $42 \%$ y a un gradiente de temperatura de 2 a $4{ }^{\circ} \mathrm{C}$. Fuerst-Waltl et al. (2006), también reportan en toros Simmental australianos un promedio de motilidad espermática de $66,6 \%$ y un vigor de 2,93 después de tres colecciones de semen, las cuales corresponden a el periodo de maduración espermática en el epidídimo. En las regiones con valores altos de índice de temperatura y humedad, los testículos tienden a estar más afectados, debido a los largos periodos de tiempo que pasan los animales durante el día. Las altas temperaturas provocan dificultad para disipar el calor corporal durante la noche.
El uso de modelos experimentales que ajustan la radiación y la velocidad del viento son fundamentales para gestionar la mitigación ambiental y proteger a los animales, garantizando el confort térmico (Mader et al. 2010).

\section{Daño del ADN por las especies reactivas de oxígeno}

Los radicales libres tienen la capacidad de dañar directamente el ADN del esperma al atacar las bases púricas y pirimidicas y el esqueleto de la desoxirribosa. Normalmente, el ADN del esperma está estrechamente empaquetado por protaminas que lo protegen del ataque de los radicales libres. Sin embargo, los machos infértiles a menudo muestran deficiencias de protaminación, dejando el ADN del esperma particularmente vulnerable al ataque de las ERO (Oliva, 2006). Alternativamente, los radicales libres pueden iniciar la apoptosis dentro del esperma, que conduce a la degradación enzimática del ADN mediado por las caspasas (Tabla 1) (Kao et al. 2008).

Tabla 1. Algunos de los daños a los espermatozoides provocados por las ERO.

\begin{tabular}{|l|l|l|}
\hline \multicolumn{1}{|c|}{ Especies } & \multicolumn{1}{c|}{ Alteración } & \multicolumn{1}{c|}{ Autor } \\
\hline Toro & Disfunción mitocondrial. & Oliveira et al. (2014) \\
\hline Carnero & Fragmentación del ADN. & Salces-Ortiz et al. (2015) \\
\hline Macho cabrio & Lipoperoxidación. & El-Tohamy et al. (2012) \\
\hline Búfalo & $\begin{array}{l}\text { Disminución de la capacidad antioxidante y su } \\
\text { relación con la LPO. }\end{array}$ & Kadirvel et al. (2014) \\
\hline $\begin{array}{l}\text { Nelore }(\text { Bos } \\
\text { indicus) }\end{array}$ & $\begin{array}{l}\text { Más resistencia al calor y menos producción de } \\
\text { ERO. }\end{array}$ & Nichi et al. (2006) \\
\hline $\begin{array}{l}\text { Brahaman (Bos } \\
\text { indicus) }\end{array}$ & $\begin{array}{l}\text { Efecto negativo en la mortalidad espermática } \\
\text { durante el proceso de sexado. }\end{array}$ & López et al. (2015) \\
\hline
\end{tabular}

Existen dos factores por los cuales el material genético está protegido del daño oxidativo: empaquetamiento muy característico del ADN, y los antioxidantes presentes en el plasma seminal. Los antioxidantes se clasifican en enzimáticos y no enzimáticos. Su mecanismo de acción es mediante la dependencia de iones y metales $(\mathrm{Cu}, \mathrm{Mn}, \mathrm{Se}$ y $\mathrm{Zn})$ como el superóxido dismutasa (SOD), catalasa (CAT), glutatión peroxidasa (GPx) y glutatión reductasa $(\mathrm{GR})$, mientas que el control no enzimático incluye a los antioxidantes como el glutatión, ácido ascórbico y ácido úrico, así como compuestos hidrosolubles y la vitamina $\mathrm{E}$ y los carotenos como liposolubles. El mecanismo de los antioxidantes se clasifica en tres vías; defensas primarias (previenen la formación de radicales libres), defensas secundarias (inactivan o barren los radicales libres) y defensas terciarias (reparan el daño oxidativo en el ADN) (Gutteridge y Michell, 1999; Perumal, 2014). 
Estudios en los que los espermatozoides fueron expuestos a ERO artificialmente, resulto en un aumento significativo de daño al ADN, causando modificaciones a las bases, produciendo deleciones, cambios en el marco de lectura y reordenamientos cromosómicos. El estrés oxidativo también se ha correlacionado con alta frecuencia de rompimientos de las cadenas dobles y sencillas del ADN (El-Tohamy, 2012).

La confirmación de la relación entre el estrés oxidativo y el daño al ADN de los espermatozoides se ha realizado mediante diversas técnicas como; marcaje de los cortes finales con dUTP mediado por la deoxinucleotidil transferasa terminal (TUNEL), ensayo de la estructura de la cromatina espermática (SCSA) y determinación de subproductos de la oxidación del ADN, 8-hidroxideoxiguanosina (8-OHdG), (Tremellen, 2008).

En un trabajo reciente se ha observado que la fragmentación del $\mathrm{ADN}$ del esperma de carnero aumenta a una temperatura de 30 ${ }^{\circ} \mathrm{C}$ y a un índice de temperatura-humedad $>$ 22 , en algunas etapas de la espermatogénesis en los animales que llevan el genotipo $\mathrm{GG}_{-660}$ de la -660G/C SNP (polimorfismo de un solo nucleótido) (Rs397514116). Como el genotipo GG. ${ }_{660}$ ha sido asociado con niveles bajos de expresión las proteínas de choque calórico HSP90AA1 en condiciones de estrés calórico, se ha sugerido que cantidades subóptimas de ARNm de HSP90AA1 provocan en el ADN de estos animales mayor susceptibilidad a ser fragmentado (SalcesOrtiz et al. 2015).

La evaluación anticipada del estrés oxidativo y el daño del ADN espermático haría una contribución sólida al perfil de análisis seminal estándar y se convertiría en herramientas diagnósticas para la evaluación de la infertilidad del macho, especialmente de origen idiopático, que deben tenerse en cuenta durante el estudio andrológico.

\section{Conclusiones}

El estrés térmico provoca alteraciones en el espermatozoide durante el ciclo espermático debido al estrés oxidativo. Este estrés puede ser causado por una alteración mitocondrial, además del daño en la protección antioxidante iniciado por las deficiencias de la dieta, la edad o por factores genéticos.

La susceptibilidad de los espermatozoides a los efectos del estrés calórico puede ser debida al hecho de que la meiosis implica cambios estructurales y bioquímicos muy marcados, tales como la recombinación homóloga, el montaje complejo sinaptonémico, y entrecruzamiento cromosómico a una temperatura inferior. Aunque la respuesta fisiológica y celular para tratar el estrés calórico de los testículos ha sido documentada, los mecanismos moleculares específicos en respuesta al estrés calórico aún no han sido establecidos. Por lo que el establecimiento y la validación de las ERO sobre la capacidad espermática bajo condiciones tropicales o de países con alta temperatura ambiental puede ser de suma importancia en vista de que no es una metodología que requiera equipo sofisticado para realizarla. Otro punto a considerar, es que estableciendo los valores de referencia para las ERO determinaría el nivel de antioxidantes necesarios en la alimentación para los animales bajo condiciones tropicales o de alta temperatura y de esa manera disminuir los problemas que afecta la capacidad reproductiva de los machos como: disfunción mitocondrial, la fragmentación del ADN, la lipoperoxidación, la disminución de la capacidad antioxidante, mas resistencia al calor de los espermas y el efecto negativo en los procedimientos de reproducción asistida.

\section{Bibliografía}

Abavisani A, Arshami J, Naserian AA, Sheikholeslami Kandelousi MA, Azizzadeh M. 2013. Quality of bovine chilled or frozen-thawed semen after addition of omega-3 fatty acids supplementation to extender. International Journal Fertility and Sterility 7:161-168.

Agarwal A, Nallella KP, Allamaneni SS, Said TM. 2004. Role of antioxidants in treatment of male infertility: an overview of the literature. Reproductive Biomedicine Online 8:616-827.

Agarwal A y Sekhon LH. 2010. The role of antioxidant therapy in the treatment of male infertility. Human Fertility 13:217-225.

Aitken RJ, Paterson M, Fisher H, Buckingham DW, van Duin M. 1995. Redox regulation of tyrosine phosphorylation in human spermatozoa and its role in the control of human sperm function. Journal of Cell Science 108:2017-2025.

Aitken RJ. 1995. Free radicals, lipid peroxidation and sperm function. Reproduction Fertility and Development 7:659-668. 
Aitken RJ. 1997. Molecular mechanisms regulating human sperm function. Molecular Human Reproduction $3: 169-173$

Aitken RJ, Gordon E, Harkiss D, Twigg JP, Milne P, Jennings Z, Irvine D S. 1998. Relative impact of oxidative stress on the functional competence and genomic integrity of human spermatozoa. Biology of Reproduction 59:1037-1046.

Aitken RJ y Roman SD. 2008. Antioxidant systems and oxidative stress in the testes. Oxidative Medicine and Cellular Longevity 1:15-24.

Aitken RJ y De Luliis GN. 2010. On the possible origins of DNA damage in human spermatozoa. Molecular Human Reproduction 16: 3-13.

Aitken RJ y Curry BJ. 2011. Redox regulation of human sperm function: from the physiological control of sperm capacitation to the etiology of infertility and DNA damage in the germ line. Antioxidants and Redox Signaling 14:367-381.

Aitken RJ Jones, KT, Robertson SH. 2012. Reactive Oxygen Species and Review Sperm Function-In Sickness and In Health. Journal of Andrology 33:1096-1106.

Alves MBR, de Andrade AFC, de Arruda RP, Batissaco L, Florez-Rodriguez SA, Lanconi. 2015. An Efficient Technique to Detect Sperm Reactive Oxygen Species: The CellRox Deep Red® Fluorescent Probe. Biochemistry and Physiology 4: 1-5.

-Balic I M, Milinkovic-Tur S, Samardzija M, Vince S. 2012. Effect of age and environmental factors on semen quality, glutathione peroxidase activity and oxidative parameters in Simmental bulls. Theriogenology 78:423-431.

Bansal AK, and Bilaspuri GS. 2010. Impacts of Oxidative Stress and Antioxidants on Semen Functions. Veterinary Medicine International pii: 686137. doi: 10.4061/2011/686137.

Block J, Chase CC, and Hansen PJ. 2002. Inheritance of resistance of bovine preimplantation embryos to heat shock: Relative importance of the maternal versus paternal contribution. Molecular Reproduction and Development 63:32-37.

Brito LFC, Silva AEDF, Barbosa R.T, Kastelic JP. 2004. Testicular thermoregulation in Bos indicus, crossbred and Bos taurus bulls: relationship with scrotal, testicular vascular cone and testicular morphology, and effects on semen quality and sperm production. Theriogenology 61:511-528.

Bucak MN, Tuncer PB, Sariözkan S, Ulutas PA. 2009. Comparison of the effects of glutamine and an amino acid solution on post-thawed ram sperm parameters, lipid peroxidation and anti-oxidant activities. Small Ruminant Research 81:13-17.

Castro LS, Hamilton TRS, Mendes CM, Nichi M, Bernabe VH, Visintin JA, Asuumpção. 2016. Sperm cryodamage occurs after rapid freezing phase: flow cytometry approach and antioxidant enzymes activity at different stages of cryopreservation. Journal of Animal Science and Biotechnology 7:17.

Catriona P, Teng S, Saunders PTK. 2009. A Single, Mild, Transient Scrotal Heat Stress Causes Hypoxia and Oxidative Stress in Mouse Testes, Which Induces Germ Cell Death. Biology of Reproduction 80:913-919.

Celino FT, Yamaguchi S, Miura C, Ohta T, Tozawa Y, Iwai T, Miura T. 2011. Tolerance of Spermatogonia to Oxidative Stress Is Due to High Levels of $\mathrm{Zn}$ and $\mathrm{Cu} / \mathrm{Zn}$ Superoxide Dismutase. PLoS ONE 6:1-11.

Chandra G, Aggarwal A, Singh A Singh A.K, KumarM, Kushwaha R, Singh YK. 2012. Oxidative stress in sperm biology. Agri. Reviews 33:54-61.

Chirault M, Lucas C, Goubault M, Chevrier C, Bressac C, Lécureuil C. 2015. A Combined Approach to Heat Stress Effect on Male Fertility in Nasonia vitripennis: From the Physiological Consequences on Spermatogenesis to the Reproductive Adjustment of Females Mated with Stressed Males. PLoS ONE 10:1-16.

Coulter GH. 1988. Thermography of bull testes, 12th Technical Conference of Artificial Insemination and Reproduction, Columbia, SC: National Association of Animal Breeders 58-63.

Coulter GH, Cook RB, Kastelic JP. 1997. Effects of dietary energy on scrotal surface temperature, seminal quality, and sperm production in young beef bulls. Journal of Animal Science 75:1048-1052.

De Grey ADNJ. 2003. A hypothesis for the minimal overall structure of the mammalian plasma membrane redox system. Protoplasma 221:3-9.

Du Plessis SS, Makker K, Desai NR, Agarwal A. 2008. Impact of oxidative stress on IVF. Expert Review of Obstetrics and Gynecology 3:539-554.

Du Plessis SS, McAllister DA, Luu A, Savia J, Agarwal A, Lampiao F. 2010. Effects of $\mathrm{H}_{2} \mathrm{O}_{2}$ exposure on human sperm motility parameters, reactive oxygen species levels and nitric oxide levels. Andrologia 42:206-210. 
El-Tohamy MM. 2012. The mechanisms by which Oxidative Stress and Free Radical Damage produces Male infertility. Life Science Journal 9:674-688.

El-Tohamy MM, Kotp MS, El-Nattat WS, Mohamed AH. 2012a. Semen Characteristics and Oxidative/ Antioxidati in Semen and Serum of Male Rabbits Supplemented with Antioxidants during Heat Stress. Iranian Journal of Applied Animal Science 2:175-183.

Espinosa-Cervantes R, y Córdova-Izquierdo A. 2013. Sexing sperm of domestic animals. Tropical Animal Health Production 45:1-8.

Feder ME, y Hofmann FE. 1999. Heat-shock proteins, molecular chaperones, and the stress response: Evolutionary and ecological physiology. Annual Review of Physiology 61:243-282.

Ferrara N. 2004. Vascular endothelial growth factor: basic science and clinical progress. Endocrine Reviews 25:581-611.

Ford WCL. 2004. Regulation of sperm function by reactive oxygen species. Human Reproduction Update10:387-399.

Forsythe JA, Jiang BH, Iyer NV, Agani F, Leung SW, Koos RD, Semenza GL. 1996. Activation of vascular endothelial growth factor gene transcription by hypoxia-inducible factor 1. Molecular and Cellular Biology 16:4604-4613.

Francavilla F, Santucci R, Macerola B, Ruvolo G, Romano R. 2000. Nitric Oxide Synthase Inhibition in Human Sperm Affects Sperm-Oocyte Fusion but Not Zona Pellucida Binding. Biology of Reproduction 63:425-429.

Fretz PC, and Sandlow JI. 2002. Varicocele: current concepts in pathophysiology, diagnosis, and treatment. Urology Clinics of North America 29:921-937.

Fuerst-Waltl B, Schwarzenbacher H, Perner C, Solkner J. 2006. Effects of age and environmental factors on semen production and semen quality of Austrian Simmental bulls. Animal Reproduction Science 95:27-37.

Galil KA, and Setchell BP. 1998. Effects of local heating of the testis on testicular blood flow and testosterone secretion in the rat. International Journal of Andrology 11:73-85.

Gong Y, Guo H, Zhang Z, Zhou H, Zhao R, He B. 2017. Heat Stress Reduces Sperm Motility via Activation of Glycogen Synthase Kinase-3 $\alpha$ and Inhibition of Mitochondrial Protein Import. Frontiers in Physiology 8:718. doi:10.3389/fphys.2017.00718.

Griveau JF, Renard P, Le Lannou D. 1994. An in vitro promoting role for hydrogen peroxide in human sperm capacitation. International Journal of Andrology 17: 300-307.

Gutteridge JMS y Michell J. 1999. Redox imbalance in the critically ill. British Medical Bulletin 55: 49-75.

Hamilton TRS, Mota MC, Signori de Castro ${ }^{\mathrm{L}}$ Monken de Assis ${ }^{\mathrm{P}}$, Adriano Felipe Perez SAF, de Carvalho JD Demarchi, GM, Muiño-Blanco T, Cebrián-Pérez JA, Nichi, M, Visintin JA, Ortiz D’Ávila AME. 2016. Evaluation of Lasting Effects of Heat Stress on Sperm Profile and Oxidative Status of Ram Semen and Epididymal Sperm. Oxidative Medicine and Cellular Longevity 16:1-12.

Hansen PJ. 2013. Cellular and molecular basis of therapies to ameliorate effects of heat stress on embryonic development in cattle. Animal Reproduction 10:322-333.

Henkel RR y Schill WB. 2003. Sperm preparation for ART. Reproductive Biology and Endocrinology 108:1-22.

Henkel RR. 2011. Leukocytes and oxidative stress: dilemma for sperm function and male fertility. Asian Journal of Andrology 13: 43-52.

Hu T, Zhu H, Sun W, Hao H, Zhao X, Du W, Wang Z. 2016. Sperm pretreatment with glutathione improves IVF embryos development through increasing the viability and antixidative capacity of sex-sorted and unsorted bull semen. J Integr Agric 15:2326-2335.

Jahanian E, Asadollahpour NH, Moradi KN. 2014. Influence of oxidative stress on sperm quality in animal. International Journal of Biosciences 4:10-16.

Jones R, Mann T, Sherins R. 1979. Peroxidative breakdown of phospholipids in human spermatozoa, spemicidal properties of fatty acid peroxides, and protective action of seminal plasma. Fertility and Sterility 31:531-537.

Jung A, y Schuppe HC. 2007. Influence of genital heat stress on semen quality in humans. Andrologia 39: 203-215.

Kadirvel G, Kumar S, Ghosh SK, Perumal P. 2014. Activity of antioxidative enzymes in fresh and frozen thawed buffalo (Bubalus bubalis) spermatozoa in relation to lipid peroxidation and semen quality. Asian Pacific Journal of Reproduction 3:210-217. 
Kao SH, Chao HT, Chen HW, Hwang TI, Liao TL, Wei YH. 2008. Increase of oxidative stress in human sperm with lower motility. Fertility and Sterility 89: 1183-1190.

Kashou AH, Sharma R, Agarwal A. 2013. Assessment of oxidative stress in sperm and semen. Methods Mol Biol. 927:351-61.

Li Y, Huang, Y, Piao Y, Nagaoka K, Watanabe G, Taya K, Li Ch. 2013. Protective effects of nuclear factor erythroid 2-related factor 2 on whole body heat stress-induced oxidative damage in the mouse testis. Reproductive Biology and Endocrinology 11: 1-10.

Lopez WO, Alvis-Miranda HR., Gamarra AF, Rendon B, Borda DA, Albicker, U, Fonoff ET, MartinezDiaz M. 2015. Effects of sexed semen and interactive effects on commercial in vitro embryo production when oocytes are collected from cows of Bos indicus, and Bos taurus breeding and crossbred cows of these subspecies. Animal Reproduction Science 156: 58-63.

Mader TL, Johnson LJ, Gaughant JB. 2010. A comprehensive index for assessing environmental stress in animals. Journal of Animal Science 88:2153-2165.

Makker K, Agarwal A, Sharma, R. 2009. Oxidative stress \& male infertility. Indian Journalof Medical Research 129:357-367.

Mieusset R, Bujan L, Mondina, C, Mansat A, Pontonnier F, Grandjean H. 1987. Association of scrotal hyperthermia with impaired spermatogenesis in infertile men. Fertility and Sterility. 48: 1006-1011.

Naaby-Hansen S, y Herr JC. 2010. Heat shock proteins on the human sperm surface. Journal of Reproductive Immunology 84:32-40.

Nezhad FS, Lavvaf A, Karimi S. 2013. Effect of heat stress on oxidative reactions in the sheep Sertoli cells. International Journal of Agriculture and Crop Sciences 6: 833-839.

Nichi M, Bols PEJ, Züge RM, Barnabe VH, Goovaerts IGF, Barnabe RC, Cortada, CNM. 2006. Seasonal variation in semen quality in Bos indicus and Bos taurus bulls raised under tropical conditions. Theriogenology 66: 822-828.

Oborna I, Fingerova H, Novotny J, Brezinova J, Svobodova M, Aziz N. 2009. Reactive oxygen species in human semen in relation to leukocyte contamination. Biomedical Papers Medicine Faculty University Palacky Olomouc Czech Republic 153: 53-57.

Oliva R. 2006. Protamines and male infertility. Human Reproduction Update 12: 417-435.

Oliveira MSR, Jardim BJO, Dias EA, Koetz Jr. C Ribas, PG, Peripolli, V McManus, C, Andrighetto CME, Guiselli LF. 2014. Scrotal infrared digital thermography as a predictor of seasonal effects on sperm traits in Braford bulls. International Journal Biometeorology. 2014;59: 357-364.

Paasch U, Grunewald S, Glander HJ. 2007. Sperm selection in assisted reproductive techniques. Soc Reprod Fertil Suppl 65:515-25.

Palmer NO, Bakos HW, Fullston T, Lane M. 2012. Impact of obesity on male fertility, sperm function and molecular composition. Spermatogenesis. 2012;2: 253-263.

Paul C, Teng S, Saunders PTK. 2009. A single, mild, transient scrotal heat stress causes hypoxia and oxidative stress in mouse testes, which induces germ cell death. Biology of Reproduction 80: 913-919.

Perumal, P. 2014. Effect of Superoxide Dismutase on Semen Parameters and Antioxidant Enzyme Activities of Liquid Stored $\left(5^{\circ} \mathrm{C}\right)$ Mithun (Bos frontalis) Semen. Journal of Animals, 1-7 (doi. or/101155/2014/821954).

Perumal P, Savino N, Sangma CTR, Khan M H, Ezung E, Chang S, Sangtam TZT 2017. Seasonal effect on physiological, reproductive and fertility profiles in breeding mithun bulls. Asian Pacific Journal of Reproduction 6(6):268-278

Pfeifer H, Conrad M, Roethlein D, Kyriakopoulos A, Brielmeier M, Bornkamm GW, Behne D. 2001. Identification of a specific sperm nuclei selenoenzyme necessary for protamine thiol cross-linking during sperm maturation. FASEB Journal 1:236-1238.

Ramires NC, Monteiro GA, Zanzarini DJ, Cavalcanti FM, Dell'aqua Jr. JA, Ozanan PF, Alvarenga MA. 2013. The relationships between scrotal surface temperature, age and sperm qualityin stallions. Livestock Science 157: 358-363.

Richer SC, and Ford WCL. 2001. A critical investigation of NADPH oxidase activity in human spermatozoa. Molecular Human Reproduction 7:237-244.

Ross C, Morriss A, Khairy M, Khalaf Y, Braude P, Coomarasamy A, El-Toukhy T. 2010. A systematic review of the effect of oral antioxidants on male infertility. Reproductive Biomedicine Online 20:711-723.

Salces-Ortiz J, Ramón M, González C, Pérez-Guzmán MD, Garde JJ, García-Álvarez O. 2015. Differences in the Ovine HSP90AA1 Gene Expression Rates Caused by Two Linked Polymorphisms at Its Promoter 
Affect Rams Sperm DNA Fragmentation under Environmental Heat Stress Conditions. PLoS ONE 10:1-19.

Setchell BP. 2006. The effects of heat on the testes of mammals. Animal Reproduction 3: 81-91.

Shekarriz M, Sharma RK, Thomas AJ, Agarwal A. 1995. Positive myeloperoxidase straining (Endtz test) as an indicator of excessive reactive oxygen species formation in semen. Journal of Assisted Reproduction and Genetics 12: 1-5.

Schweiki D, Itin A, Soffer D, Keshet E. 1992. Vascular endothelial growth factor induced by hypoxia may mediate hypoxia-initiated angiogenesis. Nature 359: 843-845.

Tremellen K. 2008. Oxidative stress and male infertility-a clinical perspective. Human Reproduction Update 14: 243-258.

Uysal O, and Bucak MN. 2007. Effects of oxidized glutathione, bovine serum albumin, cysteine and lycopene on the quality of frozen-thawed ram semen. Acta Veterinaria Brno 76: 383-390.

Vasan SS. 2011. Semen analysis and sperm function tests: How much to test? Indian Journal of Urology 27: 41-48.

Wechalekar H, Setchell BP, Peirce EJ, Ricci M, Leigh C, Breed WG. 2010. Whole body heat exposure induces membrane changes in spermatozoa from the cauda epididymidis of laboratory mice. Asian Journal of Andrology 12:591-598.

Wenger RH. 2002. Cellular adaptation to hypoxia: O2-sensing protein hydroxylases, hypoxia-inducible transcription factors, and O2-regulated gene expression. FASEB Journal 16:1151-1162. 